\title{
$\checkmark$ Research Square \\ Deficiency of miR-29 a/b1 Leads to Premature Aging and Dopaminergic Neuroprotection in Mice
}

xiaochen bai ( $D$ 13111520001@fudan.edu.cn )

Fudan University https://orcid.org/0000-0001-6927-2731

Jinghui Wang

Fudan University

Xiaoshuang Zhang

Fudan University

\section{Yilin Tang}

Huashan Hospital Fudan University

Linlin Han

Huashan Hospital Fudan University

\section{Rong Fang}

Fudan University

Zhaolin Liu

Fudan University

Hongtian Dong

Fudan University

Qing Li

Fudan University

Jingyu Ge

Fudan University

Yuanyuan Ma

Fudan University

Mei Yu

Fudan University

Ruilin Sun

Tongji University

Jian Wang

Huashan Hospital Fudan University

Jian Fei

Tongji University

Fang Huang

Fudan University 
Research

Keywords: Parkinson's disease, miR-29a/b1, glial cells, neuroinflammation, AMPK

Posted Date: May 10th, 2021

DOI: https://doi.org/10.21203/rs.3.rs-495801/v1

License: (c) (i) This work is licensed under a Creative Commons Attribution 4.0 International License. Read Full License 


\section{Abstract}

\section{Background}

Parkinson's disease (PD) is a neurodegenerative disorder characterized by progressive degeneration of midbrain dopaminergic neurons. The miR-29s family, including miR-29a and miR-29b1 as well as miR$29 b 2$ and miR-29c, are implicated in aging, metabolism, neuronal survival, and neurological disorders. In this study, the roles of $m i R-29 a / b 1$ in aging and PD were investigate.

\section{Methods}

miR-29a/b1 knockout mice (named as 29a KO hereafter) and their wild-type (WT) controls were used to analyze aging-related phenotypes. After challenged with the neurotoxin MPTP, dopaminergic injuries, glial activation and mouse behaviors were evaluated. Primary glial cells were further cultured to explore the underlying mechanisms. Additionally, the levels of miR-29s in the cerebrospinal fluid (CSF) of PD patients $(n=18)$ and healthy subjects $(n=17)$ were quantified.

\section{Results}

29a KO mice showed dramatic weight loss, kyphosis, along with increased and deepened wrinkles in skins, when compared with wild-type (WT) mice. Moreover, both abdominal and brown adipose tissues reduced in 29a KO mice, compared to their WT counterpart. However, in MPTP-induced PD mouse model, deficiency of miR-29a/b1 led to less severe damages of dopaminergic system and mitigated glial activation in the nigrostriatal pathway, and subsequently alleviated the motor impairment in 3-month-old mice. 8-month-old mutant mice maintained such a resistance to MPTP intoxication. Mechanistically, the deficiency of miR-29a/b-1 promoted the expression of neurotrophic factors in MPP ${ }^{+}$-treated primary mixed glia and primary astrocytes. In LPS-treated primary microglia, knockout of miR-29a/b-1 inhibited the expression of inflammatory factors, and promoted the expression of anti-inflammatory factors and neurotrophic factors. Knockout of $m i R-29 a / b 1$ gene increased the activity of AMPK and repressed NF$\mathrm{KB} / \mathrm{p} 65$ signaling in glial cells. Moreover, we found miR-29a level was increased in the CSF of patients with PD.

\section{Conclusions}

Our results suggest that 29a KO mice display the peripheral premature senility. The combined effects of less activated glial cells might contribute to the mitigated inflammatory responses and elicit the dopaminergic neuroprotection in miR-29a/b1 KO mice.

\section{Introduction}

Parkinson's disease (PD), characterized by progressive degeneration of midbrain dopaminergic neurons[1], ranks second among the most common neurodegenerative disorders[2]. Clinical motor symptoms are triggered by progressive loss of dopaminergic neurons in the substantia nigra pars 
compacta (SNpc) and consequently malnourished projection in the striatum[2]. Also, increasing evidence indicates the role of gliosis and inflammatory response mechanisms followed by dopamine neuronal loss in the pathogenesis of $\mathrm{PD}[3]$.

miRNAs, small non-coding RNA molecules with only about 21 nucleotides in length, emerged as ideal powerful candidates for genetic programing[4]. They exert complex effects on target gene expression post-transcriptionally by degrading mRNA or repressing translation through targeting 3'UTR of mRNA[5]. A diversity of miRNAs is either specifically abundant or selectively depleted in the nervous system, where they could contribute to neuronal apoptosis, axonal path finding, neural plasticity and particularly the development of neurological diseases[4, 6, 7]. Accumulated studies have pointed miRNAs dysfunction, including miR-29 family, exists in the pathology of neurodegenerative diseases[4, 6-8].

miR-29 family (miR-29s) consists of four members (miR-29a, miR-29b1, miR-29b2 and miR-29c), of which miR-29b1 and miR-29b2 share identical mature sequence[6, 8]. All the members have highly conserved mature sequences and identical seed sequences, and miR-29a/b1 and miR-29b2/c are encoded by two genomic clusters on different chromosomes [6, 8]. miR-29s, highly expressed in the brain, are implicated in aging, metabolism, neuronal survival, and neurological disorders[8-10]. Down-regulation of miR-29a/b1 was reported in neurodegenerative disorders, like Alzheimer's disease and Huntington's disease[6]. Simultaneously, our previous study revealed that miR-29s in the blood serum of patients with PD were significantly downregulated [11], but the mechanisms of miR-29s perturbations on PD progression are not clear.

In this study, by using miR-29a/b1 knockout (miR-29a KO) mice, changes in periphery were investigated. Further a subacute regimen of MPTP was applied to generate PD model. Damages of the nigrostriatal pathway, mouse behaviors and the underlying mechanisms were comprehensively assessed. Additionally, miR-29a levels were evaluated in the cerebrospinal fluid (CSF) of PD patients. miR-29a KO mice showed obvious premature aging, implied by weight loss, fat decreasing, kyphosis, muscle weakness, gait disorder, and wrinkle increasing and deepening. However, deficiency of miR-29a/b1 brought about mitigation of dopaminergic injury and glial activation, and consequently the alleviated behavioral impairments.

\section{Methods}

\section{Animals and drug treatments}

miR-29a/b1 knockout mice [12] and their wild-type littermates (Shanghai Research Center for Model Organisms, China) were bred in a room maintained at $20-22^{\circ} \mathrm{C}$ with a $12 \mathrm{~h}$ light/dark cycle and with food and water available ad libitum. All experimental protocols were approved by the Institutional Animal Care and Use Committee of Fudan University, Shanghai Medical College. All surgeries were performed under general anesthesia, and all efforts were made to minimize adverse effects. 
Mice were injected intraperitoneally with MPTP-HCl (Sigma, USA) in $0.9 \% \mathrm{NaCl}$, using a subacute dosing regimen of MPTP (20 mg/kg in normal saline, Sigma, USA) or normal saline for 5 consecutive days at 24 $\mathrm{h}$ intervals as described [13].

\section{Patient and clinical assessment}

The PD patients refrained from taking any anti-parkinsonian medications and fasted for at least $12 \mathrm{~h}$ before CSF samples were taken. Control subjects fasted for $12 \mathrm{~h}$ before CSF samples were taken. CSF was collected by standardized lumbar puncture procedures. Shipment and storage were performed according to the protocols from Parkinson Progression Marker Initiative (PPMI). CSF were aliquoted (200 $\mu \mathrm{l} /$ tube), flash frozen, and stored at $-80^{\circ} \mathrm{C}$.

\section{RNA extraction and quantitative RT-PCR}

Total RNA from the tissue was extracted using TRIzol reagent (TIANGEN, China) following the manufacturer's protocol. Reverse transcription was performed using random primers and the primers used in the qPCR are listed in Table 1. Relative expression levels were calculated using the comparative $\Delta \Delta \mathrm{Ct}$ method with $\beta$-action as the normalizing control.

\section{miRNA extraction, reverse transcription and quantitative real-time PCR}

Total RNA including miRNA from the CSF was extracted using miRNeasy Serum/Plasma Kit (Qiagen, Germany) following the manufacturer's protocol. Then, $5 \mu$ l of total RNA was reverse transcribed using a miRcute miRNA First-Strand cDNA Synthesis Kit (Tiangen, China). Subsequently, $2 \mu$ l of the product was used to detect miR29s expression by quantitative real-time PCR using a miRcute miRNA qPCR Detection kit (Tiangen, China). The PCR primer sequences were as follows: miR-29a (5'-TAGCACCATCTGAAATCGG3'); miR-29b (5'-TAGCACCATTTGAAATCAGT-3'); miR-29c (5'-TAGCACCATTTGAAATCGG-3'). Relative expression levels were calculated using the comparative $\triangle \triangle \mathrm{Ct}$ method with cel-miR-39 as the normalizing control.

\section{Protein extraction and western blot analysis}

Mouse tissues or cell pellets were lysed in protein extraction reagents supplemented with a protease inhibitor cocktail. $30 \mu \mathrm{g}$ of protein samples were separated by sodium dodecyl sulfate-polyacrylamide gels and then transferred onto polyvinylidene difluoride membranes (Millipore, USA) as described previously [14]. The primary antibodies used were as follows: mouse anti-tyrosine hydroxylase (1:1000; Sigma, USA), rabbit phospho-AMPKa (Thr172) (1:1000; Cell Signaling Technology, USA), rabbit anti-Bcl-2 (1:500; Cell Signal Technology, USA), rabbit anti-Sirt1 (1:800, Millipore, USA), rabbit anti-COX2 (1:1000; Abcam, USA), rabbit anti-GFAP (1:1000; Dako, Japan), mouse anti- NF-kB p65 (1:1000; Santa Cruz Biotechnology, USA), rabbit anti-phospho-NFKB p65 (1:1000; Cell Signaling Technology, USA), rabbit antip16 (1:800; Abcam, USA), mouse anti -p53 (1:800; Cell Signaling Technology, USA), mouse anti - actin (1:1000; Santa Cruz Biotechnology, USA).The membrane-bound proteins were detected with an Odyssey 
infrared imaging system (Li-Cor, USA). The protein levels were quantified by densitometry analysis using Quantity One 4.5.2 software (Bio-Rad, Hercules, USA).

\section{Immunohistochemical staining and immunofluorescence staining}

For immunohistochemical staining, brain sections (30 $\mu \mathrm{m}$ in thickness) were permeabilized, quenched the endogenous peroxidases with $0.1 \% \mathrm{H}_{2} \mathrm{O}_{2}$ and blocked in PBS-T ( $0.2 \%$ Triton X-100 in PBS) containing $10 \%$ goat serum and then incubated at $37^{\circ} \mathrm{C}$ for $1 \mathrm{~h}$. Then the sections were incubated with mouse antityrosine hydroxylase (1:1000; Sigma, USA), rabbit anti-GFAP (1:1000; Millipore, USA) and rabbit anti-lba1 (1:500 dilution, Wako, Japan) antibodies in PBS with $1 \%$ goat serum at $4{ }^{\circ} \mathrm{C}$ overnight. After washing, the sections were incubated with biotinylated anti-mouse or anti-rabbit secondary antibodies (1:200; Vector Laboratories, USA) at $37^{\circ} \mathrm{C}$ for 45 min and then with AB peroxidase (1:200; Vector Laboratories, USA) at $37^{\circ} \mathrm{C}$ for $45 \mathrm{~min}$. The peroxidase reaction was detected with DAB Peroxidase (HRP) Substrate Kit (Vector Laboratories, USA).

For immunofluorescence staining, brain sections were blocked in PBS-T (0.2\% Triton X-100 in PBS) containing $10 \%$ goat serum and then incubated at $4{ }^{\circ} \mathrm{C}$ overnight with the primary antibodies as follows: mouse anti-TH (1:500; Sigma, USA) and rabbit anti-lba1 (1:500; Wako, Japan), rabbit anti-GFAP (1:1000; Millipore, USA). After washing, the sections were incubated for $2 \mathrm{~h}$ at room temperature with secondary antibodies: donkey anti-mouse Alexa Fluor 594 and donkey anti-rabbit Alexa Fluor 488, or donkey antimouse Alexa Fluor 647 (1:1000; Invitrogen, USA). All sections were counterstained with DAPI (400 ng/ml, Sigma, USA) for 5 min. Images were collected using an Olympus FV1000 confocal microscope (Japan).

\section{Stereological cell counting}

The total numbers of TH-positive neurons in the SNpc were counted using the optical fractionator method on a Stereo Investigator system (Micro Brightfield, USA) attached to an Olympus microscope as previously described [15]. Briefly, one out of four $30 \mu$ m-thick sections and a total of six sections from bregma -2.80 to $-3.65 \mathrm{~mm}$ were collected. The $\mathrm{SN}$ region was delineated using a $5 \times$ objective, and the actual counting was performed under a $40 \times$ objective. Stereological counting was performed in a doubleblind fashion by two operators.

\section{Quantification of Iba1-, GFAP-positive cells}

To measure the number of Iba1-, GFAP-positive cells, we performed cell counting with modification and analyzed with Image-Pro Plus 6.0 (Media Cybernetics, USA) as previously described [16]. Briefly, in both the substantia nigra and dorsal striatum, two square $300 \mu \mathrm{m} \times 300 \mu \mathrm{m}$ frames were placed and cell somata within the confines of the frames were counted. The positive cell numbers in the frames in each brain section divided by the volume of the region produced the cell density. Measurements from six sections were averaged to obtain one value per mouse. The observer blinded to experimental groups performed the analysis. 


\section{Densitometric analysis}

Densitometric analysis of TH-positive fibers in the striatum was performed as previously described [17]. An average of six sections from bregma +1.60 to $0.00 \mathrm{~mm}$ were examined at $5 \times$ magnification with a light microscope (Leica, Germany). To determine the density of TH-immunoreactive staining in the striatum, a $700 \times 700 \mu \mathrm{m}$ frame was placed in the dorsal part of the striatum. Another $200 \times 200 \mu \mathrm{m}$ frame was placed in the corpus callosum to measure background values. The average of the background density readings from the corpus callosum was subtracted from the average of the density readings in the striatum for each section. Then, the average of all sections from each animal was calculated.

\section{HPLC}

The striatum was dissected from the brain tissue, then weighed and sonicated in $0.4 \mathrm{M} \mathrm{HClO}_{4}$ on ice. The homogenate was centrifuged at $15,294 \mathrm{~g}$ at $4{ }^{\circ} \mathrm{C}$ for $15 \mathrm{~min}$. The supernatant was removed for determining the concentration of monoamines and their metabolite using the chromatograph (ESA, Chelmsford, MA, USA) with a 5014B electrochemical detector.

\section{Behavioral tests}

\section{Rotarod test}

One day before the test, mice were given a training session the same as the test mode (4-40 rpm constant accelerating mode for $5 \mathrm{~min}$ ) on the rotarod (MED Associates, USA) three times separated by $1 \mathrm{~h}$ intervals. On the testing day, the time on the rod, with a maximum recording time of 300 seconds, was recorded. Data were collected from three trials separated by $1 \mathrm{~h}$ intervals. Then, the average time on the rod of all trials from each animal was calculated.

\section{Wire hanging test}

A $50 \mathrm{~cm}$ wide 2-mm thick metallic wire is secured to two vertical stands and the wire is maintained $35 \mathrm{~cm}$ above a layer of bedding material. Mice suspended by their forelimbs from the wire and subjected to a $180 \mathrm{sec}$ lasting hanging test. The suspended mice tended to support themselves with their hind paws to avoid falling and to walk along the wire to reach the platform. The number of falls (up to a maximum of 10 ) and reaches (up to a maximum of 10) during a period of $180 \mathrm{~s}$ were recorded. An aggregate score from the number of falls and reaches was derived using the formula: (10-falls + reaches)[18].

\section{Grid hanging test}

Mice were placed on a grid where it stood using all four limbs. Subsequently, the grid was turned upside down $35 \mathrm{~cm}$ above the home cage filled with bedding. The trial ended after a hanging time of 3 min was achieved. The latency to when the animal falls is recorded. The test was carried out three times with 10 min intervals, the final results were an average of the three trials as previously described [19]. 


\section{Catwalk}

Mice were pre-training to habituate to the CatWalk XT gait analysis system (Noldus, Netherlands). Each animal was allowed an uninterrupted crossing of the recording field of the runway (length of approximately $40 \mathrm{~cm}$ ) in both directions with three independent attempts at a $60 \%$ variation threshold. A high-speed camera carried out data acquisition and the software automatically classified the paw prints. Overall, runs for analysis were selected based on a minimum of five step cycles in the crossing field. After classification of the footprints in the CatWalk software, data were exported for external analysis using the Prism 7 software (GraphPad Software Inc., San Diego, CA, USA).

\section{Rearing test}

To assess the motor behavior, mice were placed individually in $400 \mathrm{~mL}$ glass beaker and the number of rearing events were recorded for $3 \mathrm{~min}$. The beaker was cleaned with $75 \%$ ethanol between each animal.

\section{Primary astrocyte and microglia cell cultures}

Primary glial cells were prepared from miR-29s deficient mice and their wild-type littermates at P1-P3, as described previously [20]. The brains were dissected and meninges were removed. Then brains were dissociated in $15 \mathrm{ml}$ tube with $2 \mathrm{ml}$ D-Hanks' solution. The cells were plated in $75 \mathrm{~cm}^{2}$ flask in DMEM medium containing $10 \%$ FBS. Culture media were changed $48 \mathrm{~h}$ later to complete medium and subsequently twice a week. After 2 weeks, astrocytes were separated from microglia by shaking at 200 rpm for $12 \mathrm{~h}$. Before experimental treatments, astrocytic cultures were plated in a six-well-plate at a density of $1 \times 10^{6} /$ well or in a 96-well plate at a density of $5 \times 10^{4}$. The preliminary culture of microglia cells was the same as that of primary astrocytes, but the purification of microglia cells were prepared as described previously[21]. Briefly, at day 21 in vitro, cultures were mildly trypsinized (0.0625\% trypsin in DHanks' solution) at $37^{\circ} \mathrm{C}$ for $30-40$ min. Floating cells (mainly astrocytes) were removed. Microglia cells were cultured with supernatant of mixed glia cells. Before experimental treatments, microglia were plated in a twenty-four well-plate at a density of $3 \times 10^{5} /$ well.

\section{Reactive oxygen species (ROS) assay}

Superoxide radicals were detected with the fluorescent probe dihydroethidium (DHE, Sigma Aldrich) [22]. Briefly, cells were plated in 96-well plates $\left(5 \times 10^{4}\right)$, and treated with $1 \mathrm{mM} \mathrm{MPP}^{+}$for $6 \mathrm{~h}, 12 \mathrm{~h}$ and $24 \mathrm{~h}$. Then cells were washed with PBS two times and incubated with $5 \mu \mathrm{M} \mathrm{DHE}$ in serum free medium at $37^{\circ} \mathrm{C}$ for $25 \mathrm{~min}$. Finally cells were washed and the fluorescence intensity was determined using fluorescence microplate-reader at an excitation wavelength of $485 \mathrm{~nm}$ and an emission wavelength of $512 \mathrm{~nm}$ [23].

\section{X-Ray MicroCT Scans}

A whole-body micro-computed tomography (microCT) scan was performed to visualize adipose tissue and skeleton of mice. The detailed three-dimensional images of the structure of mice were obtained by high resolution X-ray microCT scanning (Quantum FX; PerkinElmer, USA)[24]. Mice were anesthetized with 
$0.8 \%$ pentobarbital sodium (the volume was 10 times of their body weight), and put on the platform. The current and voltage were set to $74 \mu \mathrm{A}$ and $70 \mathrm{kVp}$. It took about 4 min per mouse. Image segmentation was conducted using a volume-editing tool, and volumes were quantified using the region of interest module within the software package (AnalyzeDirect, USA).

\section{Statistical analysis}

Data are presented as the means \pm SEM. Statistical analyses were performed using Prism 7 software (GraphPad Software Inc., San Diego, CA, USA). Statistical significance was determined using two-tailed unpaired Student's T-test for comparisons between two groups or Two-way ANOVA followed by LSD for comparisons among three or more groups. $P<0.05$ was considered statistically significant.

\section{Results}

\section{Accelerated aging in the periphery of miR-29a/b1 KO mice}

miR-29a/b1 knockout mice (29a KO) were constructed by the method of CRISPR-Cas9[12]. The strategy and the results of genotyping of mutant mice were shown in Supplementary figure 1. At 3 and 6 months old, the body weights of 29 a KO mice were reduced significantly compared to their wild type counterpart (Figure 1A). 6-month-old 29a KO mice developed apparent dermis thickening, along with increased and deepened wrinkles shown by hematoxylin and eosin (H\&E) staining (Figure 1B). Mouse bone and fat tissues were analyzed by X-Ray micro-computed tomography (microCT) scan. At 3 months old, 29a KO mice displayed obvious kyphosis (Figure 1C). Abdominal fat (subcutaneous fat and visceral fat together) and brown fat decreased in 3-month-old 29a KO mice compared to their wild type littermate (Figure 1D, $1 \mathrm{E})$. In brain, the transcripts of aging marker $p 21$, but not $p 53$, increased in the hippocampus of 29a KO mice at 6 months old. The expression levels of $p 21$ and P53 did not alter in the cortex of 29a KO and WT mice. In addition, p p53 and p16 proteins in the hippocampus of 29a KO mice showed no difference compared to their WT controls (Figure S2).

\section{Muscle weakness and abnormal walking in miR-29a/b1 KO mice}

Next, we evaluated whether deficiency of miR-29a/b1 led to behavioral changes. Wire hanging test and Grid hanging test were performed to measure the muscle strength. 29a KO mice gained lower scores in the Wire hanging test, indicated reduced forelimb strength (Figure 2A). In Grid hanging test, mutant mice showed shorter latency before falling compared to their WT counterparts (Figure 2B). However, there was no difference between WT and 29a KO mice in the Rotarod test (Figure 2C). Mouse gait was assessed by Catwalk XT gait analysis system. The speed and stride length of 29a KO and WT mice were close, however, the step cycle, stand and swing time were shorter and the duty cycle was significantly decreased, in mutant mice (Figure 2D).

miR-29s expression responds to neurotoxin treatment in multiple types of cells 
Primary cultured microglial cells, astrocytes and midbrain neurons were challenged with LPS (for microglia) or $\mathrm{MPP}^{+}$(for astrocytes and neurons), miR-29s expression were then evaluated. We found the expression levels of miR-29s did not change in MPP+treated midbrain neurons. However, all three members of miR-29s were upregulated in $\mathrm{MPP}^{+}$-treated primary astrocytes. In LPS-treated primary microglia, their expression levels were downregulated, only miR-29a expression decreased significantly (Figure S3).

\section{MPTP-induced damages of the nigrostriatal pathway are alleviated in mice with miR-29a/b1 deficiency}

To address whether deficiency of miR-29a/b1 affected the progression of PD in vivo. 3 months old miR$29 a / b 1$ knockout mice and their WT littermates received five consecutive intraperitoneal injections of MPTP or saline (NS) at $24 \mathrm{~h}$ intervals. Deficiency of miR-29a/b1 had no effect on the metabolic rate of MPTP indicated by the concentration of MPP ${ }^{+}$in the striatum 90 min after MPTP exposure (Figure S4). MPTP did not alter the striatal expression of aging marker genes $P 21, P 53$ and $P a i 1$ in the two genotypes of mice (Figure S5). In MPTP-challenged mouse nigrostriatal pathway, $\mathrm{TH}^{+}$dopaminergic neurons in the substantia nigra par compacta (SNpc), $\mathrm{TH}^{+}$nerve fiber density and $\mathrm{TH}$ protein levels in the striatum all decreased dramatically (Figure 3A-C), and consequently, striatal dopamine (DA) and its metabolite DOPAC and HVA were reduced (Figure 3D). However, MPTP-induced damages of the nigrostriatal pathways in 29a KO were markedly mitigated indicated by less severe loss of dopaminergic neurons in the SNpc and dopaminergic nerve terminals in the striatum, higher striatal TH protein levels and DA concentrations, and reduced changes in the ratios of DOPAC to DA and HVA to DA (Figure 3). Notably, under physiological conditions, DOPAC itself and HVA to DA ratio were lower, NE level was higher in 29a KO mice compared to their WT counterpart. Moreover, 5-HT and its metabolite 5-HIAA did not differ between the two genotypes of mice (Figure 3D).

\section{MPTP-induced behavioral impairments are mitigated in mice with miR-29a/b1 deficiency}

The effects of miR-29a/b1 deficiency on MPTP-induced behavioral impairment were further investigated. Rearing behavior test, a measurement of spontaneous vertical activity $[25,26]$, was performed for 3 min at $48 \mathrm{~h}$ after the last MPTP injection. In the period of 1-3 min, a relatively stable period, rearing frequency was reduced in WT mice, however it did not change in 29a KO mice after MPTP exposure (Figure 3E). Likewise, in the Pole test, a classical measure for locomotor activity in PD model [2], total time was noticeably elevated in WT mice after MPTP exposure, while it did not alter in 29a KO mice compared to their normal saline controls (Figure 3F).

\section{MPTP-induced glial activation in the nigrostriatal pathway is alleviated in mice with miR-29a/b1 deficiency}

MPTP induces glial cell activation in the nigrostriatal axis, and glial cells-mediated neuroinflammation exerts an important impact on PD pathology[27]. At 3 days after MPTP administration, we assessed whether deficiency of miR-29a/b1 influenced the activation of astrocytes and microglial cells in the SNpc 
and the striatum. Astrocytes increased dramatically in the SNpc and the striatum of both WT and 29a KO mice as revealed by immunofluorescence staining of GFAP and cell counting, however, astrocytic densities were significantly reduced in MPTP-treated 29a KO mice (Figure $4 \mathrm{~A}, \mathrm{~B}$ ). Likewise, Iba $1^{+}$ microglial cells increased in the SNpc and the striatum of WT mice, and in the striatum of 29a KO mice. Microglial densities were significantly decreased in the nigrostriatal axis of MPTP-treated 29a KO mice (Figure 4C, D).

\section{MPTP-induced damages in the nigrostriatal pathway is alleviated in older miR-29a/b1 deficient mice}

Deficiency of miR-29a/b1 led to pre-mature aging and dopaminergic protection. It was interesting to test the vulnerability of older mutant mice to MPTP-induced injury. Structurally, brains of 8-months-old 29a KO mice and their WT littermate were similar (Figure S6). 3 days after MPTP administration, the striatal TH protein levels in 29a KO mice were markedly higher compared to WT mice, whereas, GFAP proteins did not alter between the two genotypes of mice (Figure S7).

\section{Effects of miR-29a/b1 deficiency in MPP+-treated primary mixed glia}

$\mathrm{MPP}^{+}$treatment increased the expression of neurotrophic factor $B D N F, G D N F$, anti-inflammatory factor TGF- $\beta 1$, and pro-inflammatory $I L-1 \beta, I L-6$ and $C O X-2$ as well, in both WT and 29 a KO primary mixed glia. At 12,24 and $36 \mathrm{~h}$ after the treatment, the increases of $B D N F$ transcripts were more dramatic in 29a KO mixed glia, also was the increase of GDNF at $24 \mathrm{~h}$, compared to WT mixed glia. The transcripts of TGF- $\beta 1$, $I L-1 \beta, I L-6$ and $C O X-2$ did not differ between the primary mixed glia of the two genotypes (Figure $5 \mathrm{~A}-\mathrm{C}$ ). By Western blot assay, we found phosphorylated-AMPK protein level in 29a KO mixed glia was upregulated after a $12 \mathrm{~h}$-treatment of $\mathrm{MPP}^{+}$compared to PBS control and MPP+-treated WT mixed glia (Figure 5D).

\section{Effects of miR-29a/b1 deficiency in MPP+-treated primary astrocytes}

Cultured primary astrocytes were tested for the ability of proliferation and migration. In the scratch assay, primary 29a KO astrocytes proliferated and migrated faster compared to WT astrocytes at $24 \mathrm{~h}$ and $48 \mathrm{~h}$ (Figure S8A). In cell viability assay, non-treated primary 29a KO astrocytes showed higher cell viability compared to WT astrocytes. After the treatment of $\mathrm{MPP}^{+}, 29 \mathrm{a} \mathrm{KO}$ and WT astrocytes exhibited higher and lower cell viability respectively compared to their non-treated control (Figure S8B). MPP ${ }^{+}$treatment upregulated ROS products in the two groups of WT and 29a KO astrocytes (Figure S8C), and increased glucose uptake in WT, but not in 29a KO astrocytes (Figure S8D).

$\mathrm{MPP}^{+}$exposure induced the expression of neurotrophic factors and inflammation-related genes in astrocytes. At 6, 12 and $24 \mathrm{~h}$ after the exposure, BDNFtranscripts increased in WT and 29a KO astrocytes. TGF- $\beta 1$ transcript levels were dramatically elevated in 29a KO astrocytes after $6 \mathrm{~h}$ and $12 \mathrm{~h}$ treatment, and in WT astrocytes after $12 \mathrm{~h}$ treatment, and IGF-1 transcript only increased in 29a KO astrocytes after $24 \mathrm{~h}$ treatment (Figure 6A, B, Figure S9A). Expression levels of $I L-1 \beta$ increased in WT astrocytes after $6 \mathrm{~h}$ and $24 \mathrm{~h}$ treatment, and in $29 \mathrm{a} \mathrm{KO}$ astrocytes after 6,12 and $24 \mathrm{~h}$ treatment. $/ L-6$ transcripts were upregulated and did not differ in the astrocytes of two genotype. iNOS transcripts increased after $24 \mathrm{~h}$ 
treatment and did not vary between WT and 29a KO astrocytes (Figure 6C, Figure S9B). TNF- $a$ and C3 transcripts did not change after MPP ${ }^{+}$treatment for $24 \mathrm{~h}$ (Figure S9B). Activated astrocytes can be further divided into two subgroups: neurotoxic A1 type and neuroprotective A2 type. Here, we found A1 marker genes H2-T23, H2-D1, Gbp2 and Ggta1, and A2 marker CD14, but not COX-2, Clcf1 and S100a10, were significantly lower in non-treated 29a KO astrocytes compared to WT control. At $6 \mathrm{~h}$ after the treatment, H2-T23 and CD14 decreased, COX-2 and Clcf1 increased, whereas H2-D1, Gbp2, Ggta1, and S100a10 did not change in WT astrocytes; H2-T23, COX-2, Clcf1 and S100a10 increased, whereas H2-D1, Gbp2, Ggta1, and $C D 14$ did not change in 29a KO astrocytes. At $12 \mathrm{~h}$ after the treatment, $C D 14$ decreased, H2-D1, Gbp2, Ggta1, COX-2, Clcf1, and S100a10 increased, whereas H2-T23 did not change in WT astrocytes; H2-T23, H2-D1, Ggta1, COX-2, Clcf1 and S100a10 increased, whereas Gbp2 and CD14 had no alteration in 29a KO astrocytes. In addition, H2-T23 transcripts were higher, whereas H2-D1 and Gbp2 transcripts were lower in 29a KO astrocytes compared to WT controls (Figure 6D, E). By western blot assay, phosphorylated-AMPK protein level was increased in 29a KO astrocytes at $6 \mathrm{~h}$ after MPP+-treatment, while phosphorylatedAMPK protein level did not change in WT astrocytes after the treatment, and Sirt1 protein levels did not alter between WT and 29a KO astrocytes (Figure 6F). Aging markers were further evaluated. P19, P21, $P 16$ and Pai1 transcript levels were increased in WT astrocytes at $24 \mathrm{~h}$ after MPP ${ }^{+}$treatment, whereas only P21 transcript, but not the other three increased in 29a KO astrocytes, and P19 and Pai1 transcript levels were even markedly lower in 29a KO astrocytes compared to WT controls (Figure S10A). Moreover, $\mathrm{Bcl}-2$ proteins did not alter in the two genotypes of primary astrocytes with or without $\mathrm{MPP}^{+}$exposure (Figure S10B).

\section{Effects of miR-29a/b1 deficiency in LPS-treated primary microglial cells}

Inflammation-provoking molecule LPS is widely used as a stimulator for microglia. In non-treated microglia, $B D N F, G D N F$ and IGF-1 transcripts were markedly increased in 29a KO microglia compared to WT control (Figure 7A, B). At $6 \mathrm{~h}$ after LPS treatment, transcripts of pro-inflammation genes $I L-1 \beta, I L-6$, $T N F-a, C O X-2$ and $i N O S$, and anti-inflammation gene $I L-10$ were increased, those of BDNF and IGF-1 were decreased in both WT and 29a KO microglia, whereas, expression levels of anti-inflammation genes $Y M 1$ and TGF- $\beta 1$ decreased, GDNF transcript did not change in WT microglia. Likewise, GDNF transcript increased, and $Y M 1$ and TGF- $\beta 1$ did not alter in 29a KO microglia after LPS challenge. Moreover, the transcripts of $B D N F, G D N F, I L-10, T G F-\beta 1$, iNOS were significantly higher, and $I L-1 \beta, I L-6, T N F-a$ and $C O X-2$ was lower in LPS-treated 29a KO microglia compared to WT control (Figure 7A-C). By western blot, phosphorylated-AMPK (p-AMPK) protein levels were markedly upregulated in 29a KO microglia compared to WT microglia at baseline and $24 \mathrm{~h}$ after LPS administration. COX-2 proteins were increased in two genotypes of microglia, however, COX-2 protein level in 29a KO microglia was obviously reduced compared to WT microglia, at $24 \mathrm{~h}$ after LPS intoxication (Figure 7D). At $60 \mathrm{~min}$ after LPS treatment, phosphorylated-p65 (p-p65) and the ratio of p-p65 to p65, but not p65, were elevated in both WT and 29a $\mathrm{KO}$ microglia, however, p-p65 and the ratio were significantly reduced in 29a KO microglia compared to WT controls (Figure 7E). Nitrite product was elevated in LPS-treated WT microglia, but not in LPS-treated 29a KO microglia (Figure 7F). 


\section{Expression of miR-29s in CSF of PD patients and healthy subjects}

Our previous study has revealed decreasing miR-29s levels in blood serum of PD patients [11]. Here through quantitative PCR, we measured miR-29s levels in the cerebrospinal fluid (CSF) of PD patients and healthy subjects. Demographic and clinical profiles of PD patients and control groups were in Table 2 . We found that miR-29a, but not miR-29b and miR-29c, was upregulated in the cerebrospinal fluid of PD patients (Figure 8).

\section{Discussion}

In this study, roles of miR-29a/b1 in aging and Parkinson's disease were investigated. miR-29 family members show increased expression in multiple tissues including brain, muscle, and liver during aging $[10,28,29]$. miR-29s upregulate p53 expression and induce cell cycle arrest [30, 31], and participate in p16/Rb-driven cellular senescence as well [32]. However, both pro-aging and anti-aging roles of miR-29s have been reported in the periphery and the central nervous system (CNS). miR-29s are induced during aging in short-lived turquoise killifish brain, where they elicit neuroprotection [33]. On the other hand, significant up-regulation of miR-29s contributes to aging-induced sarcopenia in rodents [28]. Functions of miR29s in ageing are very complex. Even within one single system, different functions of miR-29s have been explored $[34,35]$.

Dooley et al. found 10 weeks old miR-29a/b $1^{-/}$mice exhibited reduced body weights and lengths, and had less white fat compared to the control WT mice[36]. Similarly, we observed that 8 weeks old miR$29 a / b-1^{\%}$ mice were shorter compared to their WT littermate (data not shown). 29a KO mice at 3 months old showed dramatic weight loss. Moreover, their abdominal fat (subcutaneous fat and visceral fat together) and brown fat all decreased. Aging-associated kyphosis was apparent in mutant mice. Changes in skin and muscle are markers of aging [37-39]. 6-month-old 29a KO mice developed apparent thickening of dermis, along with increased and deepened wrinkles. Metalloproteinase Zmpste24-deficient mice at 16 weeks completely loss the subcutaneous fat layer and develop muscle weakness $[39,40]$. Such lipodystrophy and muscle weakness were observed in miR-29a/b1 knockout mice aged at 3 months. However, in the brains of 29a KO mice, p53 and p16 protein levels did not alter compared to their WT littermate. miR-29a/b1 deficient astrocytes even showed higher proliferation ability in vitro. Therefore, cell senescence was not obvious in the brain of miR-29a/b1 KO mice; Progeria phenotypes of mutant mice mainly displayed in the periphery. Aging is defined as a multifactorial process that affects most of the biological functions of the organism and increases susceptibility to diseases and death [10]. However, aging processes in different tissue/organs can be non-synchronized.

Both beneficial and detrimental roles of miR-29 family have been reported in the diseases of CNS. The miR-29 family was significantly decreased in neuroblastoma and neuronal cells following oxygen and glucose deprivation/reperfusion (OGD/R) treatment [41, 42], and miR-29a significantly increased in the resistant dentate gyrus, but decreased in the vulnerable CA1 region of the hippocampus after transient forebrain ischemia and short periods of reperfusion [43]. Thus, miR-29s play a protective role in ischemic 
injury. Papadopoulou et al. have reported that $m i R-29 a / b 1$ knockout mice develop a progressive disorder characterized by locomotor impairment and ataxia [8]. In this study, we found mutant mice exhibited posture instability. However, after challenged with MPTP, they showed behavioral resistance to some extent. Survival of dopaminergic neurons is regulated by glial cells. Astrocytes and microglia produce multiple neuron-supporting neurotrophic factors. Meanwhile, they are the major innate immune cells in the CNS, and involve in the progression of neuroinflammation and PD. miR-29s expression did not alter in $\mathrm{MPP}^{+}$-exposed midbrain neurons, however, $\mathrm{MPP}^{+}$induced the expression of all three members of miR-29s in primary cultured astrocytes, and miR-29s expression, especially miR-29a, was downregulated in LPStreated primary microglial cells. The results suggest that in different types of cells, the response of miR29 s to stimuli varies.

In $\mathrm{MPP}^{+}$-challenged mixed glia culture, deficiency of miR-29a/b1 increased the expression of $B D N F$ and GDNF. Moreover, $\mathrm{MPP}^{+}$enhanced the expression of IGF-1 in miR-29a mutant astrocytes, while Pai 1 transcript, a molecule involved in aging and pro-inflammation, was inhibited in MPP ${ }^{+}$-treated 29a KO astrocytes. Additionally, MPP ${ }^{+}$stimulation resulted in complex changes in A1 and A2 markers depended on the duration and markers per se. It is worthy of further studies. In primary microglial culture, deficiency of miR-29a/b1 mitigated LPS-induced inflammatory response, and simultaneously promoted the expression of anti-inflammation cytokines and neurotrophic factors. In all three types of primary glial cultures, phosphorylated AMPK protein levels were upregulated in mutant cells. Under LPS treatment, phosphorylated NF-KB p65 subunit and the ratio of p-p65 to p65 were reduced in miR-29a/b1 mutant microglial cells. Activation of AMPK can further stimulate Sirtuin 1, and indirectly inhibit NF-KB pathway and the expression of downstream inflammation-related target genes[44] AMPK activation has been reported to be neuroprotective in MPTP-induced PD mice[45]. Our experimental results suggested that knockout of $m i R-29 a / b 1$ gene increased the activity of AMPK, which might subsequently inhibit the activation of NF-KB pathway and the inflammatory responses in microglia, and might consequently inhibit the astrocyte activation. All in all, enhanced AMPK and/or reduced NF-KB p65 signaling might contribute to the milder inflammation response in miR-29a/b1 mutant glia cells and the protection of nigrostriatal axis in miR-29a/b1 deficient mice (Figure S11).

miR-29s family is highly expressed in brain $[8,10]$. We found that in the CSF of patients with PD, miR-29a level was significantly elevated compared to healthy subjects, whereas in our previous studies, the serum levels of miR-29s were markedly downregulated in PD patients [11], and miR-29s were associated with cognitive impairment in PD as well [46]; As we know, changes in miRNAs in the cerebrospinal fluid (CSF) and blood serum are associated with neurological diseases. However, miRNA expression profile of CSF is substantially different from that of serum [47]. Differential levels of the same miRNAs are detected in these two fluids under the conditions of diseases. For examples, in the CSF of glioma patients, exosomal miR-21 levels were significantly higher than in the controls; whereas there was no difference of exosomal miR-21 expression in the serum [48]. The differences of serum and CSF miR-29s levels might be attributed to the different origination, dysfunction of the blood-CSF barrier, dysregulation of miRNA transportation, and worthy of further studies. 


\section{Conclusions}

The present study shows that deficiency of miR-29a/b1 leads to pre-mature aging in the periphery, however such mutation maintains mouse brain in a low-inflammatory microenvironment, and elicits certain resistance to the dopaminergic neurotoxin in adult and older mice.

\section{List Of Abbreviations}

PD: Parkinson's disease

29a KO $₫ i R-29 a / b 1$ knockout mice

WT: wild-type

CSF: cerebrospinal fluid

SNpc: substantia nigra pars compacta

AMPK: adenosine monophosphate-activated protein kinase

miR-29s: miR-29 family

TH: tyrosine hydroxylase

ROS: reactive oxygen species

BDNF: brain-derived neurotrophic factor

COX-2: cyclooxygenase-2

GDNF: glial-cell line-derived neurotrophic factor

GFAP: glial fibrillary acidic protein

Iba-1: lonized calcium binding adapter molecule 1

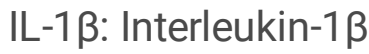

IL-6: Interleukin-6

LPS: Lipopolysaccharide

MPTP: 1-Methyl-4-phenyl-1,2,3,6-tetrahydropyridine

p-AMPK: Phosphorylated-AMP-activated protein kinase

Sirt 1: Sirtuin 1 


\section{Declarations}

\section{Ethics approval and consent to participate}

All animal experimental protocols were approved by the Institutional Animal Care and Use Committee of Fudan University, Shanghai Medical College.

All participants provided written informed consent in accordance with the Declaration of Helsinki. The study was approved by the Human Studies Institutional Review Board, Huashan Hospital, Fudan University.

\section{Consent for publication}

Not applicable

\section{Availability of data and materials}

All data generated or analysed during this study are included in this published article and its supplementary information files.

\section{Competing interests}

The authors declare that they have no competing interests.

\section{Authors' contributions}

$\mathrm{FH}, \mathrm{JF}, \mathrm{JW}$ and RLS proposed and supervised the study. FH, JF, JW, RLS, XCB, JHW, XSZ designed research studies, acquired data, analyzed data and wrote the manuscript. YLT and LLH contributed to the sample collection and clinical characterization of the patients and analyzed data. XCB, JHW, XSZ, RF, ZLL, HTD, QL, JYG, MY and YYM conducted experiments. All authors contributed to the interpretation of data and the revision of the manuscript.

\section{Funding and Acknowledgements}

This work was supported by grants from the National Natural Science Foundation of China (31970908, 31671043, 81261120568 and 91949118), Shanghai Municipal Science and Technology Major Project (No.2018SHZDZX01, No. 2017SHZDZX01) and ZJLab, the Science and Technology Commission of Shanghai Municipality (18DZ2290700), the innovative research team of high-level local university in Shanghai, FDUROP and the Open Project of State Key Laboratory of Medical Neurobiology (SKLMN2003). The authors are grateful to the study participants.

\section{References}


1. Kikuchi, T., et al., Human iPS cell-derived dopaminergic neurons function in a primate Parkinson's disease model. Nature, 2017. 548(7669): p. 592-596.1. Kikuchi, T., et al., Human iPS cell-derived dopaminergic neurons function in a primate Parkinson's disease model. Nature, 2017. 548(7669): p. 592-596.

2. Kam, T.I., et al., Poly(ADP-ribose) drives pathologic alpha-synuclein neurodegeneration in Parkinson's disease. Science, 2018. 362(6414).

3. Martinez, B. and P.V. Peplow, MicroRNAs in Parkinson's disease and emerging therapeutic targets. Neural Regen Res, 2017. 12(12): p. 1945-1959.

4. Kosik, K.S., The neuronal microRNA system. Nat Rev Neurosci, 2006. 7(12): p. 911-20.

5. Selbach, M., et al., Widespread changes in protein synthesis induced by microRNAs. Nature, 2008. 455(7209): p. 58-63.

6. Roshan, R., et al., Brain-specific knockdown of miR-29 results in neuronal cell death and ataxia in mice. RNA, 2014. 20(8): p. 1287-97.

7. Hebert, S.S., et al., Loss of microRNA cluster miR-29a/b-1 in sporadic Alzheimer's disease correlates with increased BACE1/beta-secretase expression. Proc Natl Acad Sci U S A, 2008. 105(17): p. 641520.

8. Papadopoulou, A.S., et al., Deficiency of the miR-29a/b-1 cluster leads to ataxic features and cerebellar alterations in mice. Neurobiol Dis, 2015. 73: p. 275-88.

9. Caravia, X.M., et al., The microRNA-29/PGC1alpha regulatory axis is critical for metabolic control of cardiac function. PLoS Biol, 2018. 16(10): p. e2006247.

10. Ugalde, A.P., et al., Aging and chronic DNA damage response activate a regulatory pathway involving miR-29 and p53. EMBO J, 2011. 30(11): p. 2219-32.

11. Bai, X., et al., Downregulation of blood serum microRNA 29 family in patients with Parkinson's disease. Sci Rep, 2017. 7(1): p. 5411.

12. Liao, Y., et al., Pravastatin regulates host foreign-body reaction to polyetheretherketone implants via miR-29ab1-mediated SLIT3 upregulation. Biomaterials, 2019. 203: p. 12-22.

13. Selvaraj, S., et al., Neurotoxin-induced ER stress in mouse dopaminergic neurons involves downregulation of TRPC1 and inhibition of AKT/mTOR signaling. J Clin Invest, 2012. 122(4): p. 1354-67.

14. Wang, Z., et al., Pro-survival and anti-inflammatory roles of NF-KB c-Rel in the Parkinson's disease models. Redox Biol, 2020. 30: p. 101427.

15. Liberatore, G.T., et al., Inducible nitric oxide synthase stimulates dopaminergic neurodegeneration in the MPTP model of Parkinson disease. Nat Med, 1999. 5(12): p. 1403-9.

16. Baiguera, C., et al., Late-onset Parkinsonism in NFkappaB/c-Rel-deficient mice. Brain, 2012. 135(Pt 9): p. 2750-65.

17. Huang, D., et al., Long-term Changes in the Nigrostriatal Pathway in the MPTP Mouse Model of Parkinson's Disease. Neuroscience, 2018. 369: p. 303-313. 
18. van Putten M., A.-R.A., Louvain-la-Neuve L., The use of hanging wire tests to monitor muscle strength and condition over time. Treat-Nmd., August 4,2011. Eu 1-12: p. Available online at http://www.treatnmd.eu/downloads/file/sops/dmd/MDX/DMD_M.2.1.004.pdf.

19. van Putten, M., et al., Comparison of skeletal muscle pathology and motor function of dystrophin and utrophin deficient mouse strains. Neuromuscul Disord, 2012. 22(5): p. 406-17.

20. Shao, W., et al., Suppression of neuroinflammation by astrocytic dopamine D2 receptors via alphaBcrystallin. Nature, 2013. 494(7435): p. 90-4.

21. Saura, J., J.M. Tusell, and J. Serratosa, High-yield isolation of murine microglia by mild trypsinization. Glia, 2003. 44(3): p. 183-9.

22. Passagne, I., et al., Implication of oxidative stress in size-dependent toxicity of silica nanoparticles in kidney cells. Toxicology, 2012. 299(2-3): p. 112-24.

23. Lee, Y.J., et al., Inhibitory effect of a tyrosine-fructose Maillard reaction product, 2,4-bis(phydroxyphenyl)-2-butenal on amyloid-beta generation and inflammatory reactions via inhibition of NF-kappaB and STAT3 activation in cultured astrocytes and microglial BV-2 cells. J Neuroinflammation, 2011. 8: p. 132.

24. Liu, X.J., et al., Characterization of a murine nonalcoholic steatohepatitis model induced by high fat high calorie diet plus fructose and glucose in drinking water. Lab Invest, 2018. 98(9): p. 1184-1199.

25. Chinta, S.J., et al., Cellular Senescence Is Induced by the Environmental Neurotoxin Paraquat and Contributes to Neuropathology Linked to Parkinson's Disease. Cell Rep, 2018. 22(4): p. 930-940.

26. Willard, A.M., R.S. Bouchard, and A.H. Gittis, Differential degradation of motor deficits during gradual dopamine depletion with 6-hydroxydopamine in mice. Neuroscience, 2015. 301: p. 254-67.

27. Huang, D., et al., Dynamic Changes in the Nigrostriatal Pathway in the MPTP Mouse Model of Parkinson's Disease. Parkinson's Disease, 2017. 2017: p. 1-7.

28. Hu, Z., et al., MicroRNA-29 induces cellular senescence in aging muscle through multiple signaling pathways. Aging (Albany NY), 2014. 6(3): p. 160-75.

29. Fenn, A.M., et al., Increased micro-RNA $29 \mathrm{~b}$ in the aged brain correlates with the reduction of insulinlike growth factor-1 and fractalkine ligand. Neurobiol Aging, 2013. 34(12): p. 2748-58.

30. Varela, I., et al., Accelerated ageing in mice deficient in Zmpste24 protease is linked to p53 signalling activation. Nature, 2005. 437(7058): p. 564-8.

31. Park, S.Y., et al., miR-29 miRNAs activate $p 53$ by targeting p85 alpha and CDC42. Nat Struct Mol Biol, 2009. 16(1): p. 23-9.

32. Martinez, I., et al., miR-29 and miR-30 regulate $B-M y b$ expression during cellular senescence. Proc Natl Acad Sci U S A, 2011. 108(2): p. 522-7.

33. Ripa, R., et al., MicroRNA miR-29 controls a compensatory response to limit neuronal iron accumulation during adult life and aging. BMC Biol, 2017. 15(1): p. 9.

34. Boon, R.A., et al., MicroRNA-29 in aortic dilation: implications for aneurysm formation. Circ Res, 2011. 109(10): p. 1115-9. 
35. Heid, J., et al., Age-dependent increase of oxidative stress regulates microRNA-29 family preserving cardiac health. Sci Rep, 2017. 7(1): p. 16839.

36. Dooley, J., et al., The microRNA-29 Family Dictates the Balance Between Homeostatic and Pathological Glucose Handling in Diabetes and Obesity. Diabetes, 2016. 65(1): p. 53-61.

37. Liu, Y., et al., Genome-wide scan identified genetic variants associated with skin aging in a Chinese female population. J Dermatol Sci, 2019. 96(1): p. 42-49.

38. Ahmed, I.A., et al., Natural anti-aging skincare: role and potential. Biogerontology, 2020.

39. Fong, L.G., et al., Heterozygosity for Lmna deficiency eliminates the progeria-like phenotypes in Zmpste24-deficient mice. Proc Natl Acad Sci U S A, 2004. 101(52): p. 18111-6.

40. Pendas, A.M., et al., Defective prelamin A processing and muscular and adipocyte alterations in Zmpste24 metalloproteinase-deficient mice. Nat Genet, 2002. 31(1): p. 94-9.

41. Wei, R., et al., MiR-29 Targets PUMA to Suppress Oxygen and Glucose Deprivation/Reperfusion (OGD/R)-induced Cell Death in Hippocampal Neurons. Curr Neurovasc Res, 2018. 15(1): p. 47-54.

42. Cao, L., et al., MicroRNA-29b alleviates oxygen and glucose deprivation/reperfusion-induced injury via inhibition of the p53-dependent apoptosis pathway in N2a neuroblastoma cells. Exp Ther Med, 2018. 15(1): p. 67-74.

43. Ouyang, Y.B., et al., Astrocyte-enriched miR-29a targets PUMA and reduces neuronal vulnerability to forebrain ischemia. Glia, 2013. 61(11): p. 1784-94.

44. Salminen, A., J.M. Hyttinen, and K. Kaarniranta, AMP-activated protein kinase inhibits NF-KB signaling and inflammation: impact on healthspan and lifespan. J Mol Med (Berl), 2011. 89(7): p. 667-76.

45. Lu, M., et al., Metformin Prevents Dopaminergic Neuron Death in MPTP/P-Induced Mouse Model of Parkinson's Disease via Autophagy and Mitochondrial ROS Clearance. Int J Neuropsychopharmacol, 2016. 19(9).

46. Han, L., et al., Association of the serum microRNA-29 family with cognitive impairment in Parkinson's disease. Aging (Albany NY), 2020. 12(13): p. 13518-13528.

47. Burgos, K.L., et al., Identification of extracellular miRNA in human cerebrospinal fluid by nextgeneration sequencing. RNA, 2013. 19(5): p. 712-22.

48. Shi, R., et al., Exosomal levels of miRNA-21 from cerebrospinal fluids associated with poor prognosis and tumor recurrence of glioma patients. Oncotarget, 2015. 6(29): p. 26971-81.

\section{Tables}

Table 1. Primers for qPCR analysis. 


\begin{tabular}{|c|c|}
\hline Name & Sequence $\left(\begin{array}{ll}5^{\prime} & 3^{\prime}\end{array}\right)$ \\
\hline Mouse actin F & CAGGATGCAGAAGGAGATTAC \\
\hline Mouse actin $\mathrm{R}$ & AACGCAGCTCAGTAACAGTC \\
\hline Mouse BDNF F & TCATACTTCGGTTGCATGAAGG \\
\hline Mouse BDNF R & AGACCTCTCGAACCTGCCC \\
\hline Mouse CD14 F & GGACTGATCTCAGCCCTCTG \\
\hline Mouse CD14 R & GCTTCAGCCCAGTGAAAGAC \\
\hline Mouse Clcf1 F & CTTCAATCCTCCTCGACTGG \\
\hline Mouse Clcf1 R & TACGTCGGAGTTCAGCTGTG \\
\hline Mouse COX2 F & GTTCATCCCTGACCCCCAAG \\
\hline Mouse COX2 R & ACTCTGTTGTGCTCCCGAAG \\
\hline Mouse GDNF F & GACGTCATGGATTTTATTCAAGCCACC \\
\hline Mouse GDNF R & CTGGCCTACTTTGTCACTTGTTAGCCT \\
\hline Mouse Gbp2 F & GGGGTCACTGTCTGACCACT \\
\hline Mouse Gbp2 R & GGGAAACCTGGGATGAGATT \\
\hline Mouse Ggta1 F & GTGAACAGCATGAGGGGTTT \\
\hline Mouse Ggta1 R & GTTTTGTTGCCTCTGGGTGT \\
\hline Mouse H2-D1 F & TCCGAGATTGTAAAGCGTGAAGA \\
\hline Mouse H2-D1 R & ACAGGGCAGTGCAGGGATAG \\
\hline Mouse H2-T23 F & GGACCGCGAATGACATAGC \\
\hline Mouse H2-T23 R & GCACCTCAGGGTGACTTCAT \\
\hline Mouse IGF-1 F & AGAGCCTGCGCAATGGAATAAAGT \\
\hline Mouse IGF-1 R & TTGGTGGGCAGGGATAATGAGG \\
\hline Mouse IL-1 F & GCAACTGTTCCTGAACTC \\
\hline Mouse IL-1 $\beta$ R & CTCGGAGCCTGTAGTGCA \\
\hline Mouse IL-6 F & CATAGCTACCTGGAGTACATGA \\
\hline Mouse IL-6 R & CATTCATATTGTCAGTTCTTCG \\
\hline Mouse IL-10 F & AGCCGGGAAGACAATAACTG \\
\hline Mouse IL-10 R & GGAGTCGGTTAGCAGTATGTTG \\
\hline
\end{tabular}

Page 20/30 


\begin{tabular}{|ll|} 
Mouse iNOS F & CCCTTCCGAAGTTTCTGGCAGCAGC \\
\hline Mouse iNOS R & GGCTGTCAGAGCCTCGTGGCTTTGG \\
\hline Mouse p21 F & GTGGGTCTGACTCCAGCCC \\
\hline Mouse p19Arf $\mathrm{F}$ & GCCGCACCGGAATCCT \\
\hline Mouse p19Arf $\mathrm{R}$ & TTGAGCAGAAGAGCTGCTACGT \\
\hline Mouse Pai1 F & TCAGAGCAACAAGTTCAACTACACTGAG \\
\hline Mouse Pai1 R & CCCACTGTCAAGGCTCCATCACTTGCCCA \\
\hline Mouse p53 F & GAGTATACCACCATCCACTACAAG \\
\hline Mouse p53 R & GCACAAACACGAACCTCAAAG \\
\hline Mouse S100a10 F & CCTCTGGCTGTGGACAAAAT \\
\hline Mouse S100a10 R & CTGCTCACAAGAAGCAGTGG \\
\hline Mouse Slc10a6 F & GCTTCGGTGGTATGATGCTT \\
\hline Mouse Slc10a6 R & CCACAGGCTTTTCTGGTGAT \\
\hline Mouse TGF- $\beta 1 \mathrm{~F}$ & CCTGAGTGGCTGTCTTTTGA \\
\hline Mouse TGF- $\beta 1$ R & CGTGGAGTTTGTTATCTTTGCTG \\
\hline Mouse TNF-a F & CACGCTCTTCTGTCTACTGAACTTC \\
\hline Mouse TNF-a R & GCAGCCTTGTCCCTTGAAGAGAACC \\
\hline Mouse YM1 F & GTCACAGGTCTGGCAATTC \\
\hline Mouse YM1 R & GTAGAGACCATGGCACTG \\
\hline
\end{tabular}

Table 2. Demographic and clinical profiles of PD patients and control groups. 


\begin{tabular}{lccccc}
\hline & Controls & PD & Hoehn \& Yahr & Hoehn \& Yahr & Hoehn \& Yahr \\
& & & stage I & stage II & stage III \\
\hline No. of subjects & 17 & 18 & 5 & 11 & 2 \\
Age, $y$ & $61.88 \pm 6.314$ & $61.22 \pm 6.44$ & $62.2 \pm 4.207$ & $60.09 \pm 7.648$ & 65 \\
F/M & $9 / 8$ & $10 / 8$ & $4 / 1$ & $5 / 6$ & $1 / 1$ \\
Disease & & $38.83 \pm 42.02$ & $34.4 \pm 16.09$ & $42.18 \pm 51.64$ & $31.5 \pm 44.55$ \\
duration, mo & & & & & \\
MMSE & & $23.89 \pm 5.04$ & $21.4 \pm 5.505$ & $26.36 \pm 2.501$ & $16.5 \pm 6.364$ \\
\hline
\end{tabular}

Abbreviations: $P D=$ Parkinson's disease; $m o$ =month; MMSE =Mini Mental State Examination. The data are presented as means $\pm S D$.

Figures 
A

Weight

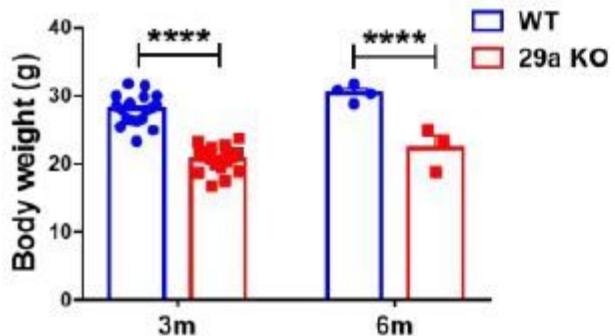

C

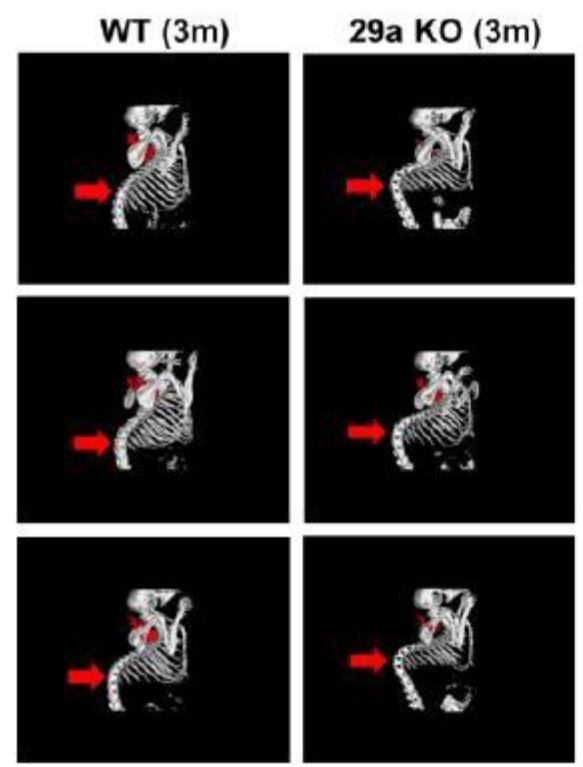

B

WT $(6 \mathrm{~m})$

29a KO (6m)

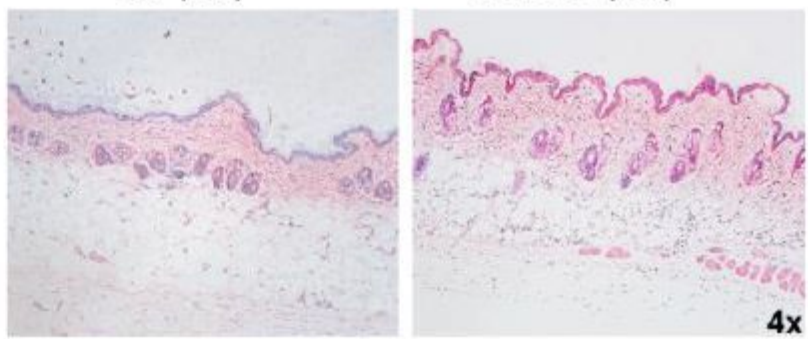

D

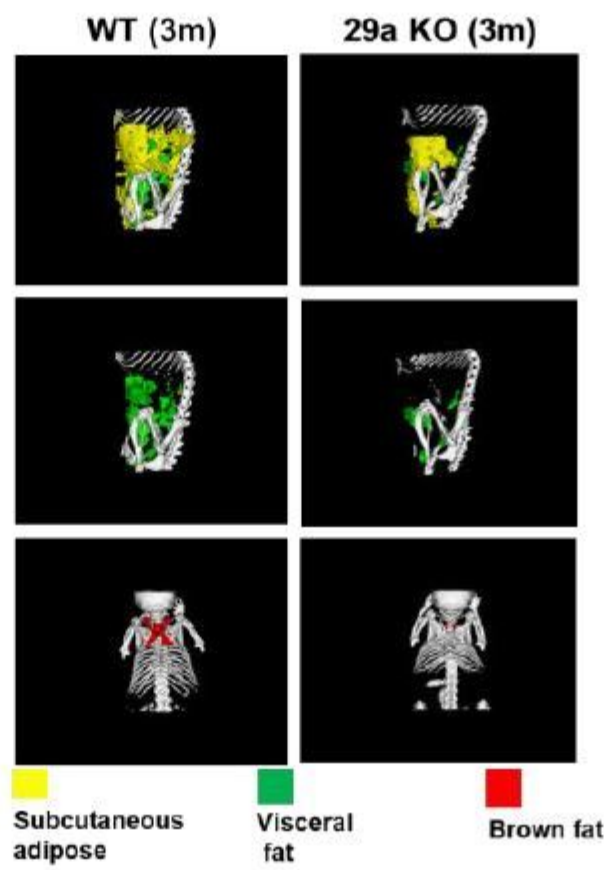

$\mathbf{E}$
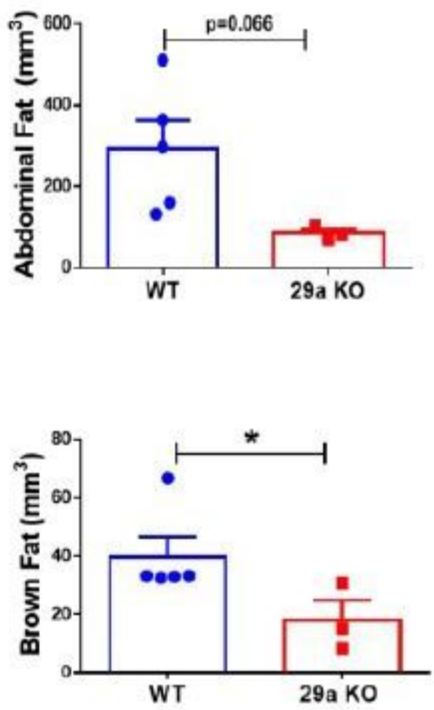

Figure 1

Peripheral characteristics of miR-29a KO mice. 
A

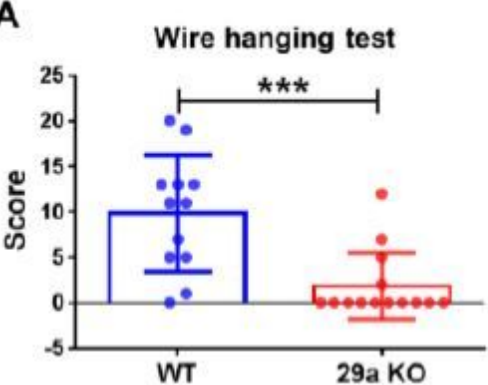

D
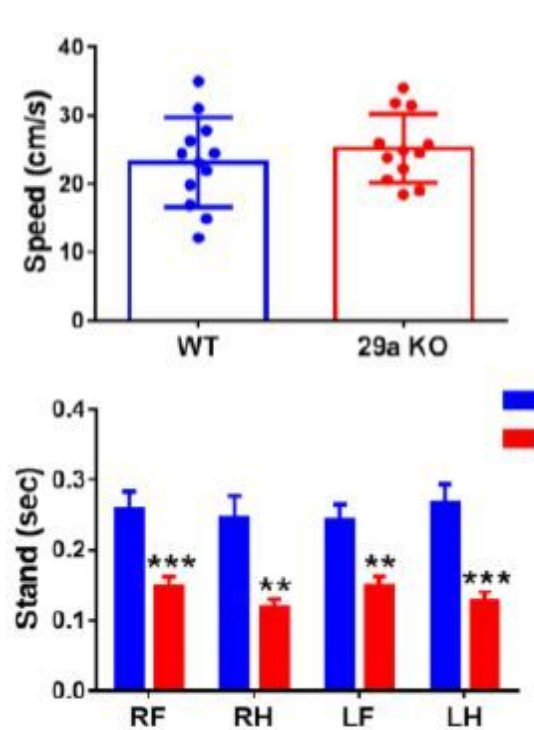

B

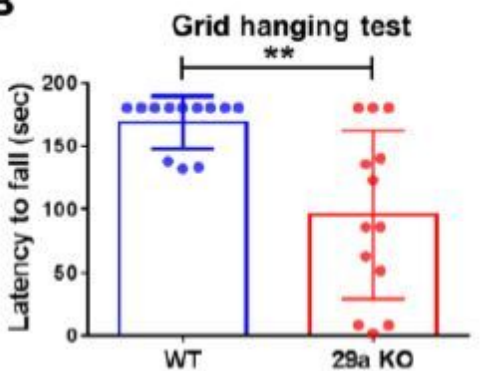

C

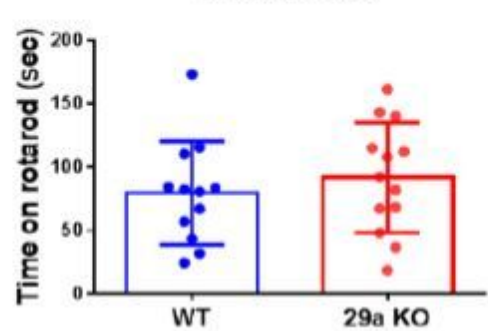

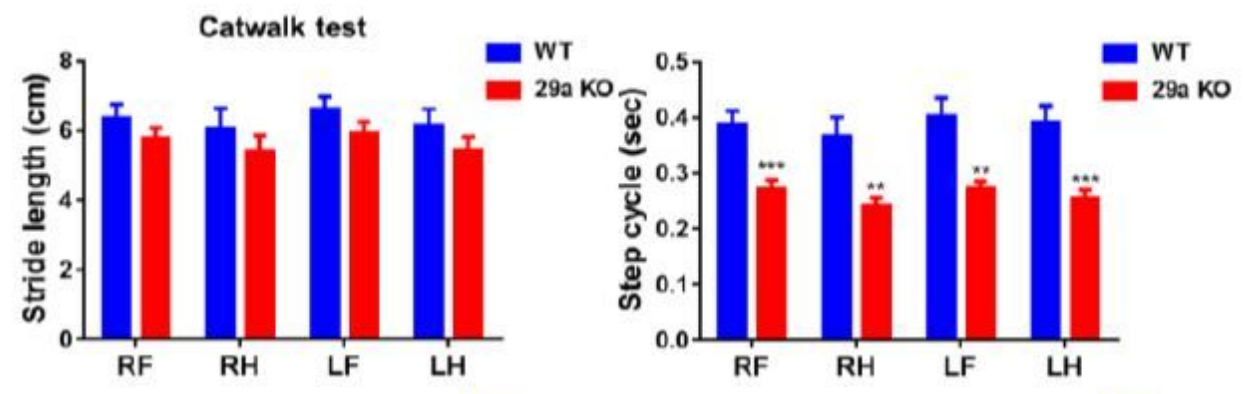

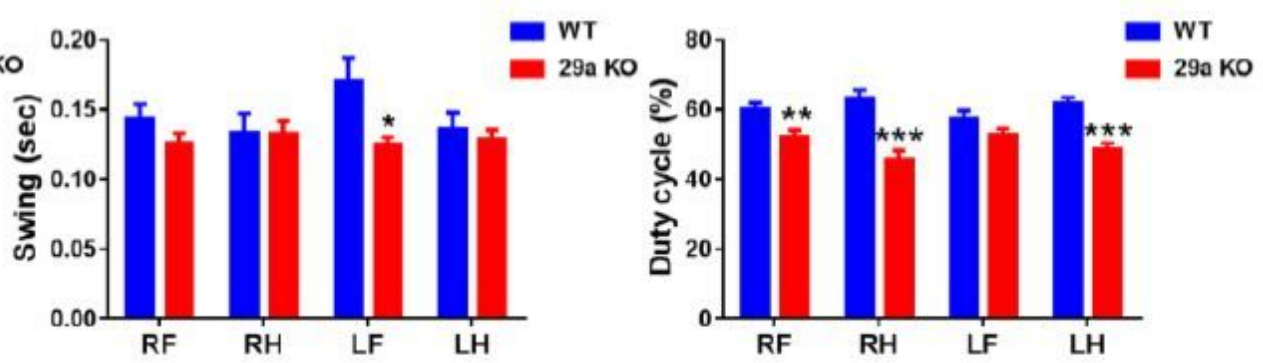

Figure 2

Muscle weakness and abnormal walking in miR-29a/b1 KO mice. 
A

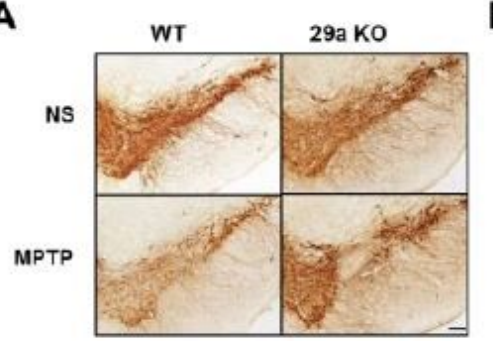

B
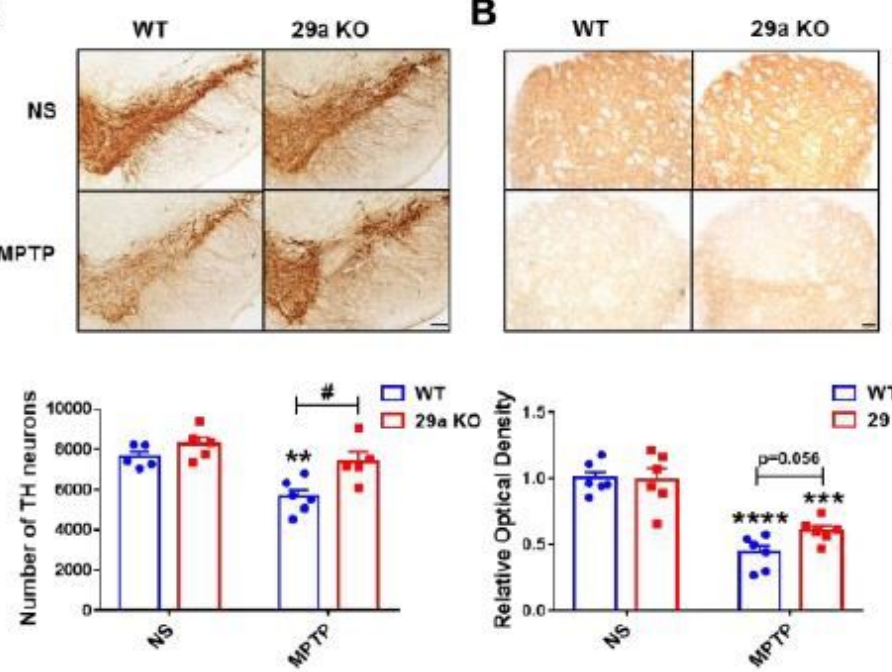

C
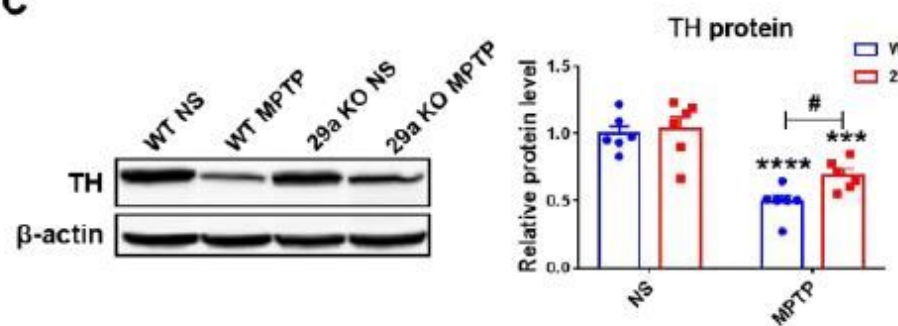

$\mathbf{E}$

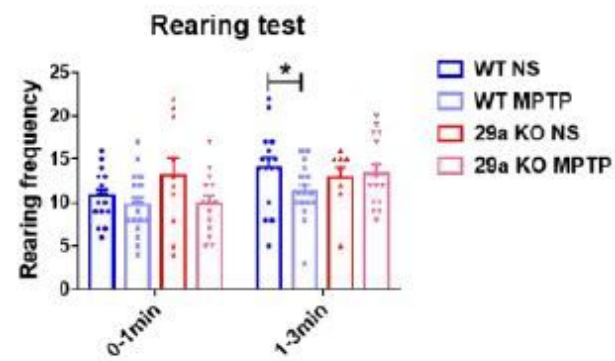

D
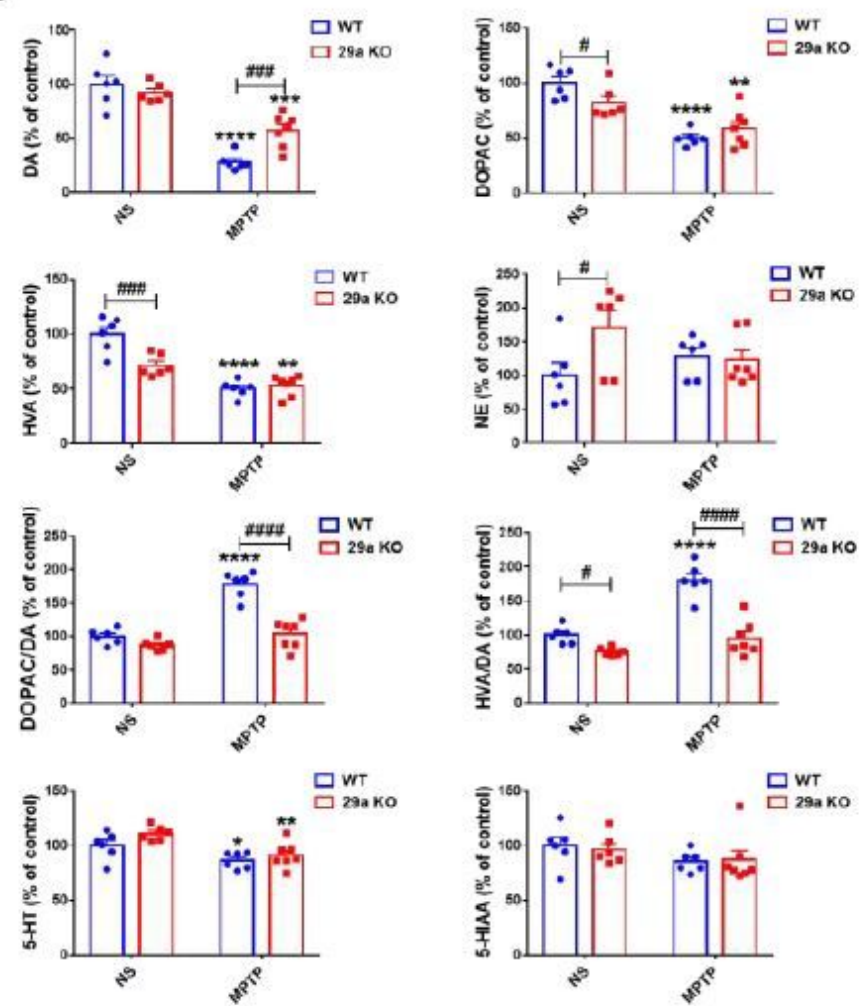

$\mathbf{F}$

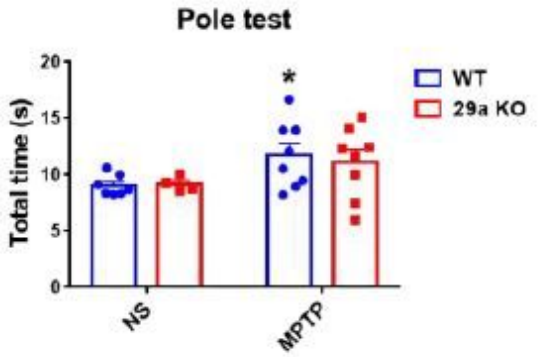

\section{Figure 3}

Analysis of the nigrostriatal pathway and behavioral performance of WT and miR-29a/b1 KO mice at 3 days after MPTP administration. 
A

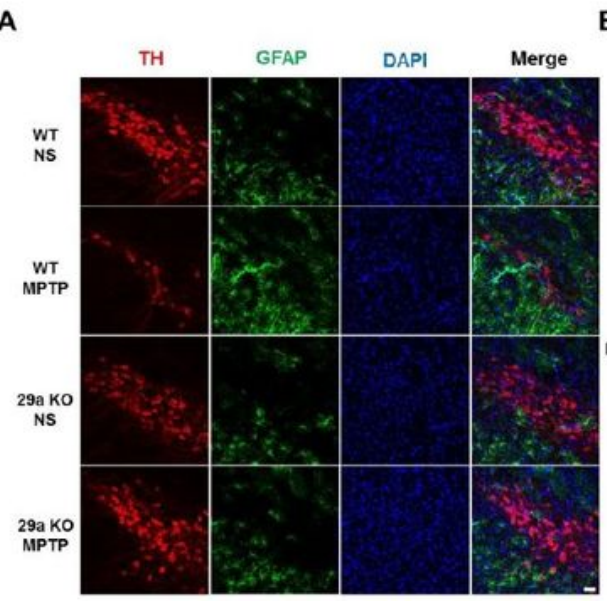

B
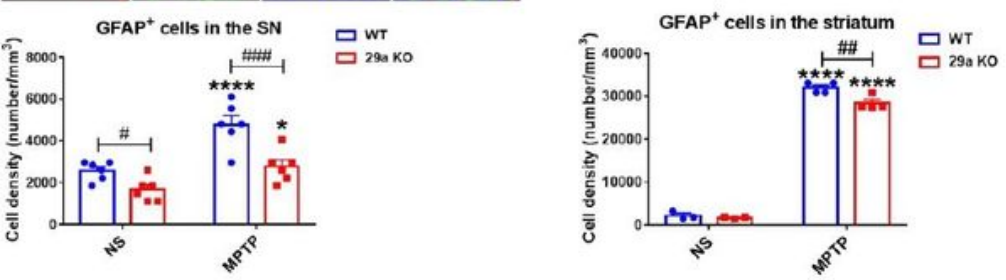

c
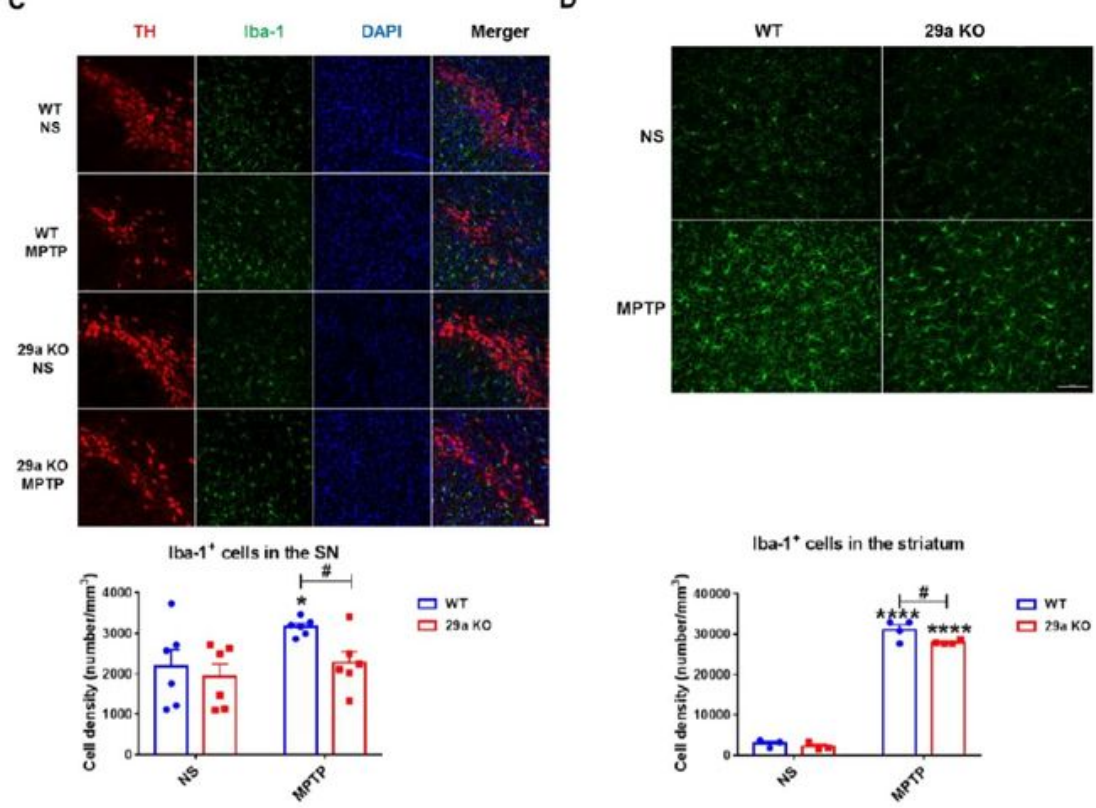

Figure 4

Analysis of glial activation in the nigrostriatal pathway at 3 days after MPTP administration. 
A

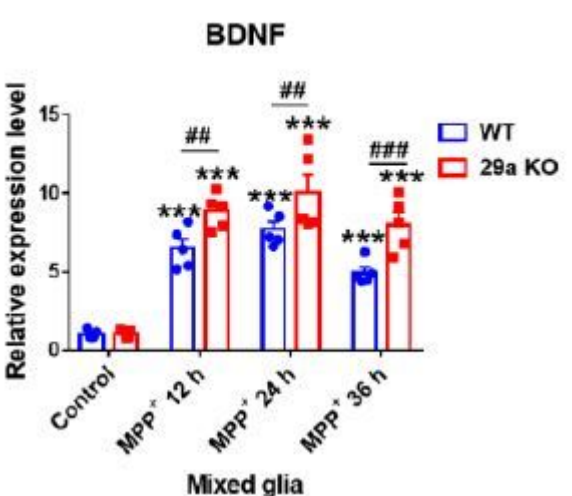

C

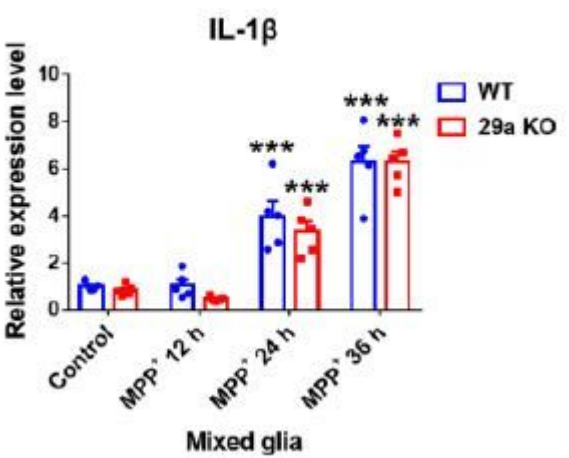

D

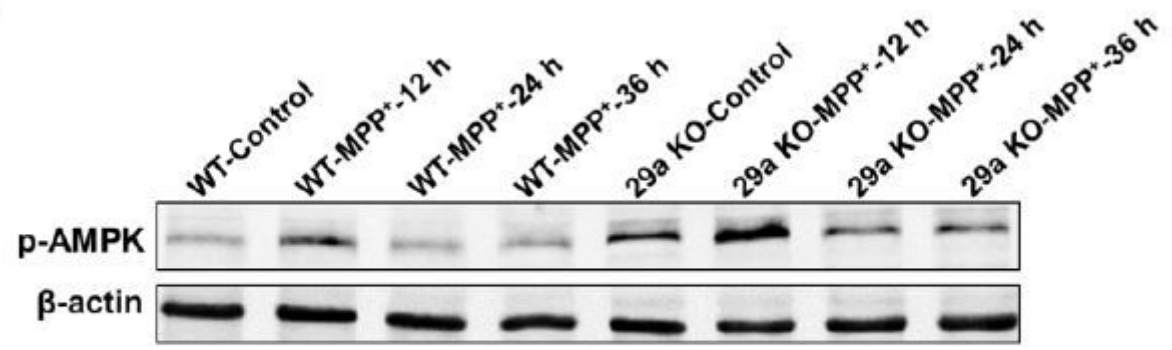

IL-6

Mixed glia
B
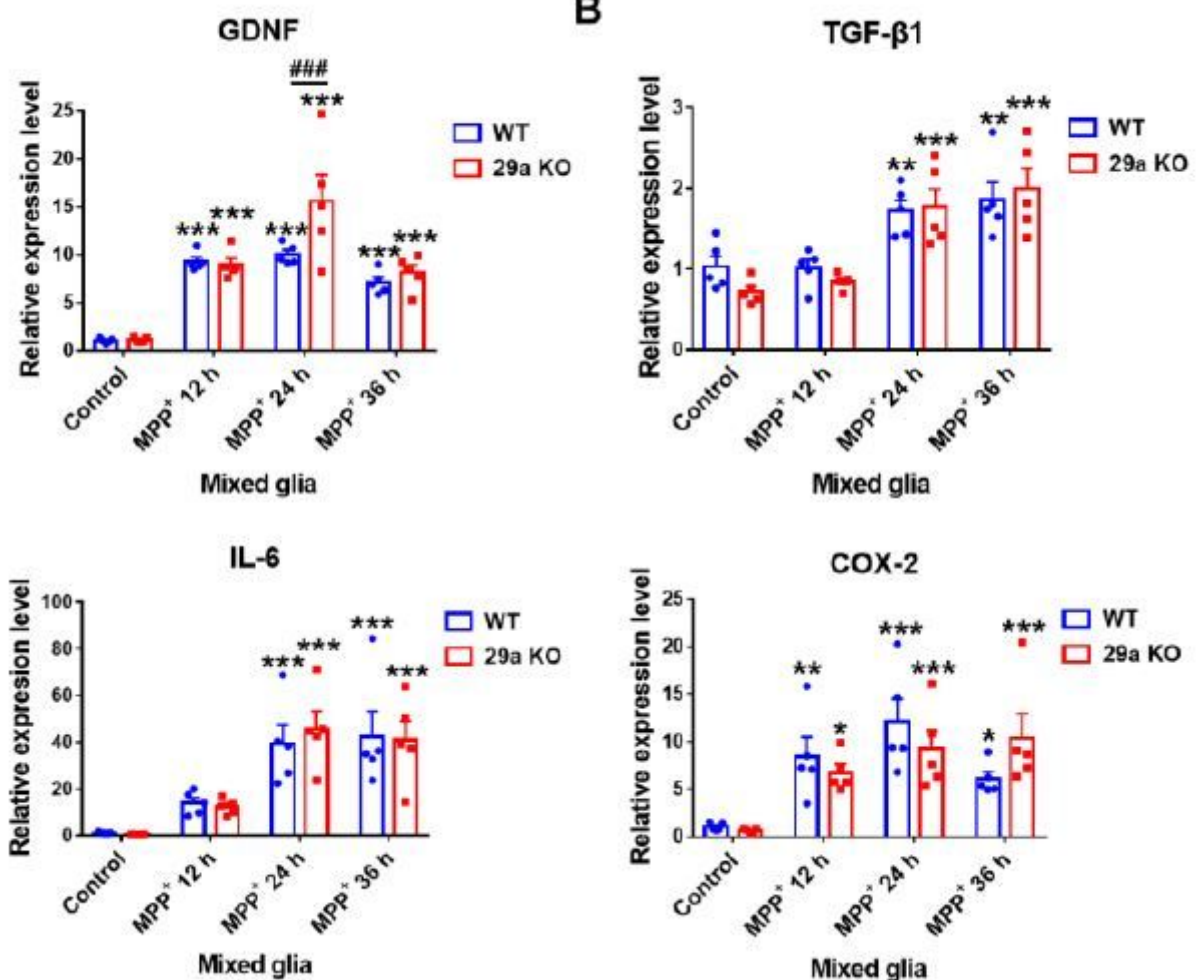

Mixed glia
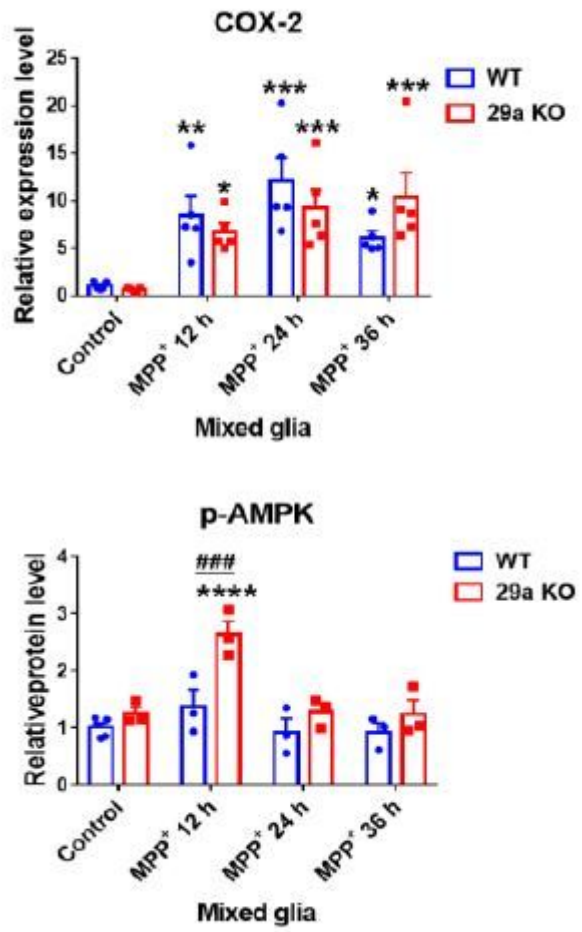

Figure 5

Effects of miR-29a/b1 deficiency in MPP+-treated primary mixed glia. 

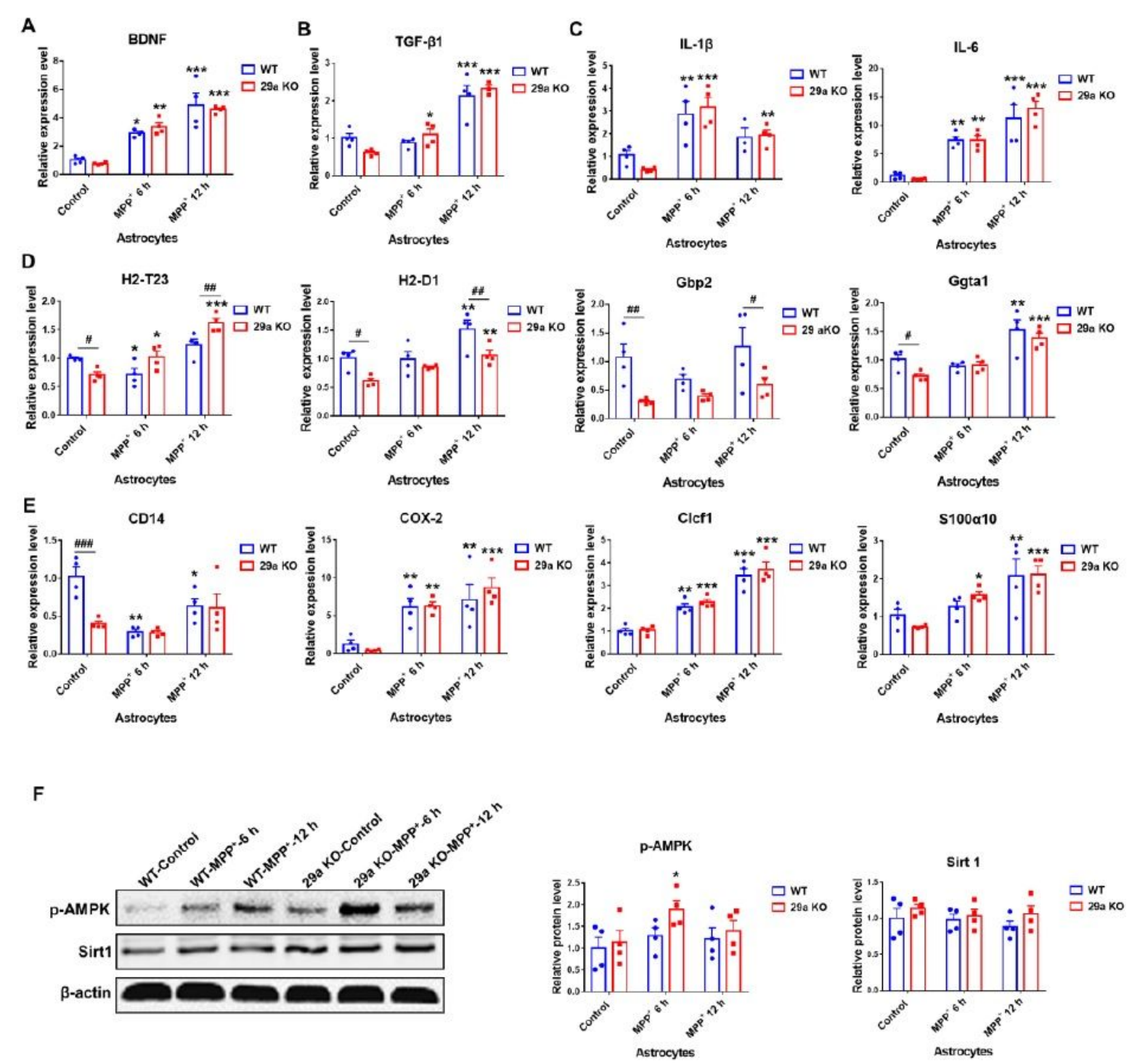

Figure 6

Effects of miR-29a/b1 deficiency in toxin-treated primary astrocytes. 


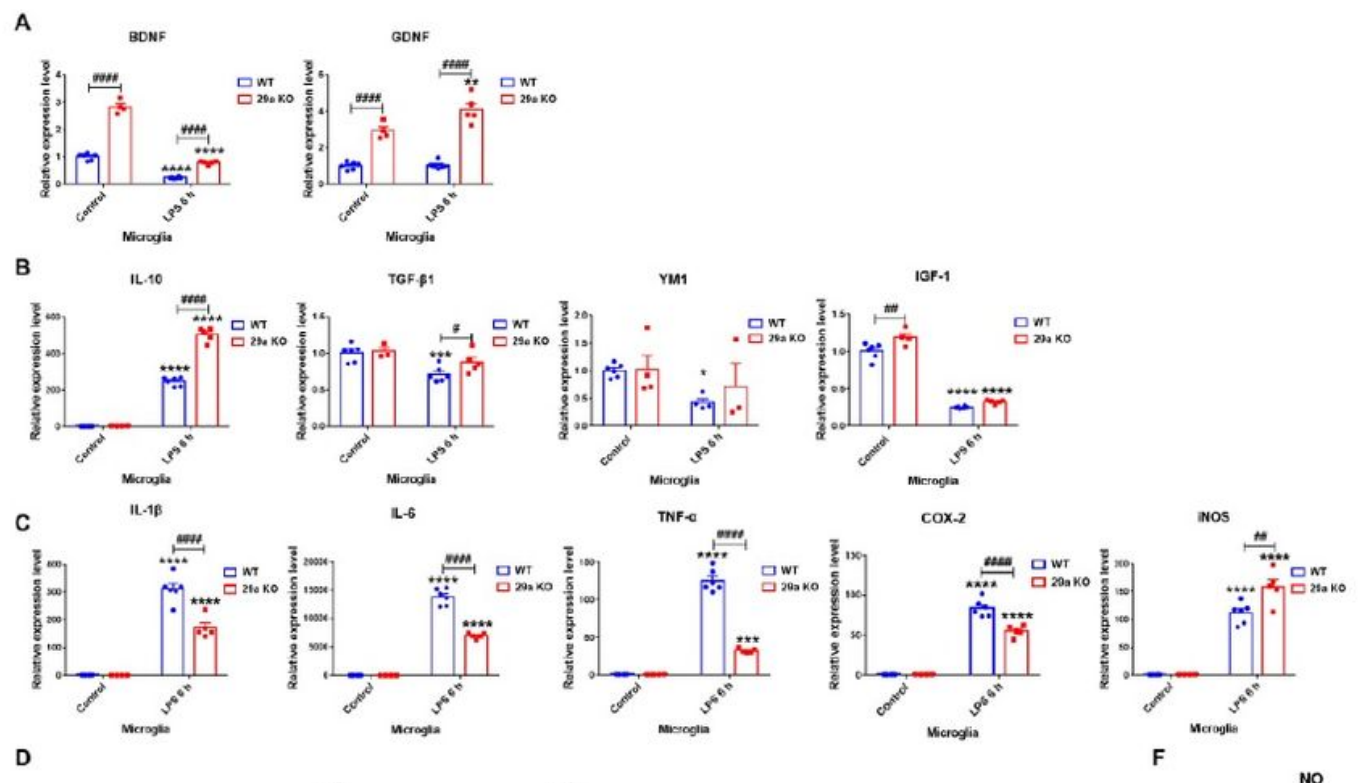

D
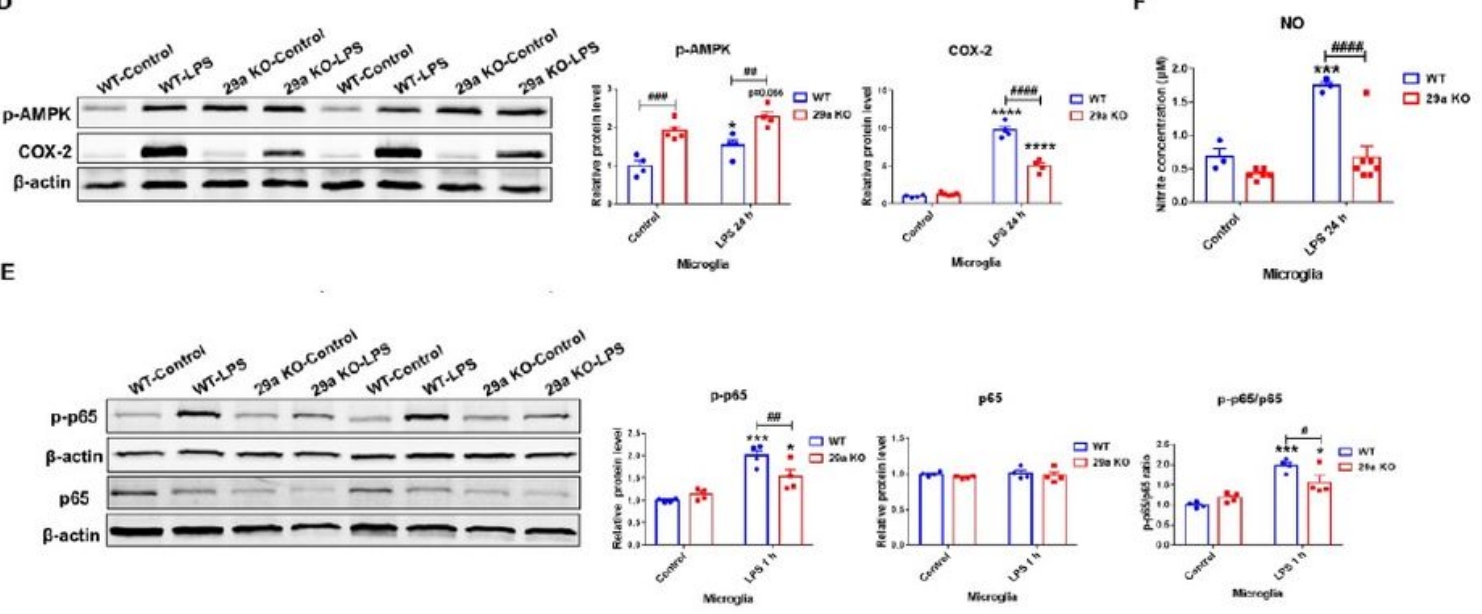

Figure 7

Effects of miR-29a/b1 deficiency in MPP+-treated primary microglia. 

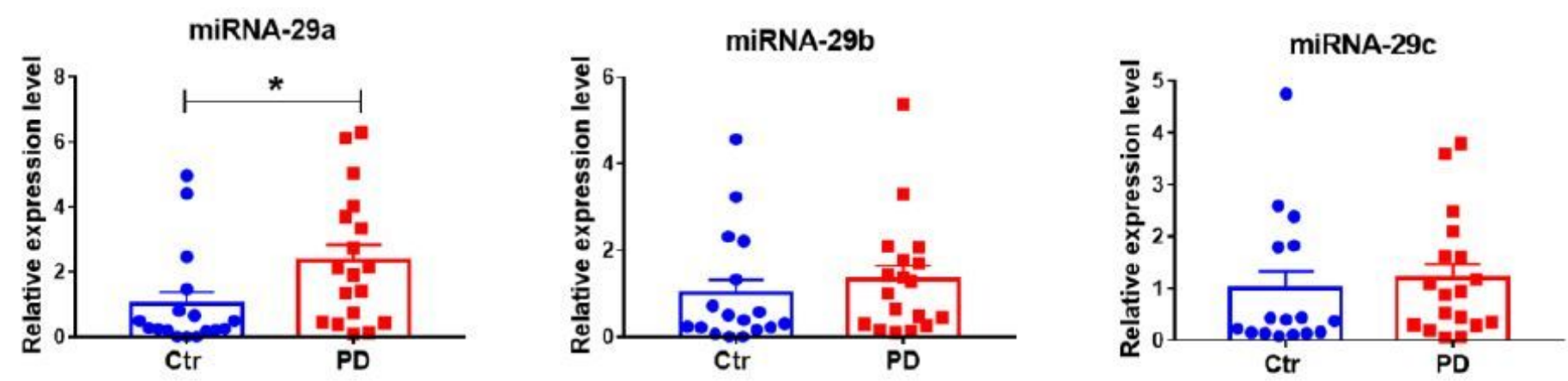

Figure 8

The expression levels of miR-29s in the cerebrospinal fluid of control subjects and patients with PD and in the serum of PD mice.

\section{Supplementary Files}

This is a list of supplementary files associated with this preprint. Click to download.

- Supplementarylnformation.pdf 